\title{
Total Haemolytic Complement Activity at Diagnosis as an Indicator of the Baseline Activity of Antineutrophil Cytoplasmic Antibody-associated Vasculitis
}

\author{
Jung Yoon Pyo, M.D. ${ }^{1}$, Lucy Eunju Lee, M.D., Ph.D. ${ }^{1}$, Sung Soo Ahn, M.D. ${ }^{1}$, Jason Jungsik Song, M.D., Ph.D. ${ }^{1,2}$, \\ Yong-Beom Park, M.D., Ph.D. ${ }^{1,2}$, Sang-Won Lee, M.D., Ph.D. ${ }^{1,2}$ \\ ${ }^{1}$ Division of Rheumatology, Department of Internal Medicine, Yonsei University College of Medicine, ${ }^{2}$ Institute for Immunology and \\ Immunological Diseases, Yonsei University College of Medicine, Seoul, Korea
}

\begin{abstract}
Objective. The total haemolytic complement activity $(\mathrm{CH} 50)$ assay evaluates the functioning of the complement system. Accumulating evidence indicates that the activation of the complement system plays a critical role in the pathogenesis of antineutrophil cytoplasmic antibody-associated vasculitis (AAV). Therefore, this study aimed to investigate whether $\mathrm{CH} 50$ levels at diagnosis could reflect the baseline activity of AAV. Methods. This retrospective study included 101 immunosuppressive drug-naïve patients with AAV. At diagnosis, all patients underwent clinical assessments for disease activity, including measurement of the Birmingham Vasculitis Activity Score (BVAS) and Five Factor Score (FFS), and laboratory evaluations, such as tests for $\mathrm{CH} 50, \mathrm{C} 3$, and $\mathrm{C} 4$ levels. The association between $\mathrm{CH} 50$ levels and disease activity was determined. Results. The median BVAS and FFS at diagnosis were 12.0 and 1.0, respectively, whereas the median $\mathrm{CH} 50$ level was $60.4 \mathrm{U} / \mathrm{mL}$. There was a negative correlation between the $\mathrm{CH} 50$ level and BVAS $(r=-0.241 ; p=0.015)$. A CH50 cut-off value of $62.1 \mathrm{U} / \mathrm{mL}$ was used to classify the patients into two groups: patients with $\mathrm{CH} 50$ levels $<62.1 \mathrm{U} / \mathrm{mL}$ (low-CH50 group) and those with $\mathrm{CH} 50$ levels $\geq$ $62.1 \mathrm{U} / \mathrm{mL}$ (high-CH50 group). The low- $\mathrm{CH} 50$ group had a higher proportion of patients with high disease activity, based on the BVAS, than the high-CH50 group (52.5\% vs. 23.8\%, p=0.004). Additionally, the low-CH50 group exhibited a lower relapse-free survival rate than the high- $\mathrm{CH} 50$ group; however, this difference was not statistically significant $(p=0.082)$. Conclusion. Low $\mathrm{CH} 50$ levels at diagnosis may reflect high baseline activity of AAV. (J Rheum Dis 2021;28:85-93)
\end{abstract}

Key Words. Antineutrophil cytoplasmic antibody, Vasculitis, Activity, Complement hemolytic activity assay

\section{INTRODUCTION}

Antineutrophil cytoplasmic antibody (ANCA)-associated vasculitis (AAV) is characterised by necrotising vasculitis that affects small vessels, including arterioles, venules, and capillaries. The 2012 Chapel Hill Consensus Conference Nomenclature of the Vasculitides (the 2012 CHCC definitions) and the 2007 European Medicines Agency algorithm (the 2007 EMA algorithm) indicated that AAV consists of three subtypes: microscopic polyangiitis (MPA), granulomatosis with polyangiitis (GPA), and eosinophilic granulomatosis with polyangiitis
(EGPA) [1,2]. Although various immune mechanisms are involved in its pathogenesis, the alternative complement pathway is central to the acceleration and maintenance of the vicious inflammatory cycle of AAV $[3,4]$. Therefore, with the progression of inflammation, the consumption of complement components may be theoretically increased, resulting in hypocomplementemia.

The total haemolytic complement activity ( $\mathrm{CH} 50$ ) assay is a widely used assay to evaluate abnormalities of all complement components ( $\mathrm{C} 1$ to $\mathrm{C} 9$ ). $\mathrm{CH} 50$ can be reduced owing to congenital complement deficiencies, increased complement consumption, or decreased comple-

Received : December 15, 2020, Revised : January 11, 2021, Accepted : January 14, 2021

Corresponding to : Sang-Won Lee (i) http://orcid.org/0000-0002-8038-3341

Division of Rheumatology, Department of Internal Medicine, Yonsei University College of Medicine, 50-1 Yonsei-ro, Seodaemun-gu, Seoul 03722, Korea. E-mail : sangwonlee@yuhs.ac 
ment synthesis [5]. In the pathogenesis of AAV, the alternative complement system is activated and generates C5a, which attracts and prime neutrophils [6]. Considering that $\mathrm{CH} 50$ assesses the overall activity of the whole complement system, and that hypocomplementemia has been reported in patients with vasculitis and associated with the patient prognosis [7-10], we aimed to investigate whether $\mathrm{CH} 50$ at diagnosis reflects the baseline activity and predicts disease outcomes during follow-up in immunosuppressive drug-naïve AAV patients in this study.

\section{MATERIALS AND METHODS}

\section{Patients}

We included and reviewed the medical records of 101 immunosuppressive drug-naïve patients with AAV who were evaluated at the tertiary medical center, between October 2000 and December 2019. All patients were classified or reclassified to have MPA, GPA, or EGPA based on the 2007 EMA algorithm and the 2012 CHCC definition $[1,2]$. All patients had well-documented medical records to obtain clinical and laboratory results and to calculate the Birmingham Vasculitis Activity Score (BVAS) and the Five Factor Score (FFS) at the time of diagnosis $[11,12]$. Additionally, all patient records had information for both tests for ANCA and complements, such as $\mathrm{CH} 50, \mathrm{C} 3$, and C4 $[13,14]$, and all patients had been followed up for at least 3 months. None of the patients had any serious medical condition, such as malignancies and serious infections, at the time of diagnosis, and they had never received any immunosuppressive drug until diagnosis. This study was approved by the Institutional Review Board of Severance Hospital (4-2017-0673). The need for written informed consent was waived owing to the retrospective nature of this study.

\section{Clinical data at diagnosis and during follow-up}

Regarding variables at diagnosis, information on age and sex was collected as demographic data. Clinical data, such as those on AAV subtypes, and those pertaining to disease activity, including the BVAS and FFS, were collected. Laboratory data, including those of white blood cell and platelet counts, haemoglobin, fasting glucose, blood urea nitrogen (BUN), creatinine, total serum protein, serum albumin, alkaline phosphatase (ALP), aspartate aminotransferase, alanine aminotransferase, total bilirubin, erythrocyte sedimentation rate (ESR), and C-reactive protein (CRP), were obtained. In addition, ANCA and CH50 data were collected. Data related to poor prognosis outcomes, including all-cause mortality, relapse, and end-stage renal disease (ESRD), were evaluated. Relapse was defined as recurrence or new onset disease requiring an increase in glucocorticoids and switching the immunosuppressant due to increased disease activity. ESRD was defined as a medical condition requiring renal replacement therapy for $>3$ months. The follow-up duration was defined as the interval between the date of the diagnosis of AAV and date of the last visit for living patients. For deceased patients, it was defined as the interval between the date of diagnosis of AAV and the date of death, whereas for patients who had other poor outcomes, it was defined as the interval starting from the date of diagnosis of AAV until the date of occurrence of the first poor outcome.

\section{Classification of variables: Tertiles based on the BVAS and two groups based on $\mathrm{CH} 50$}

Since there is no clear BVAS criterion to define high disease activity, we divided the baseline BVAS into tertiles: high activity, intermediate activity, and low activity. We defined baseline high disease activity as a BVAS $\geq 13$, which is the highest tertile in our data. Furthermore, we categorised the patients with AAV into two groups based on the calculated optimal cut-off of $\mathrm{CH} 50$ for high baseline activity: patients with $\mathrm{CH} 50$ levels below the optimal cut-off were classified into the low-CH50 group, and those with $\mathrm{CH} 50$ levels equal to or above the cut-off were classified into the high-CH50 group.

\section{Statistical analyses}

All statistical analyses were conducted using SPSS software (version 23 for Windows; IBM Corp., Armonk, NY, USA). Continuous variables were expressed as the median and interquartile range, whereas categorical variables were demonstrated as frequencies and percentages. The correlation coefficient was obtained using Pearson correlation analysis. The optimal cut-off was extrapolated by calculating the receiver operator characteristic curve and selecting the maximised sum of sensitivity and specificity. The relative risk (RR) was analysed using contingency tables and the chi-square test. The standardised correlation coefficient was assessed by multivariable linear regression analysis using variables showing significant differences in univariable analysis. The cumulative survival rates between the groups were compared using KaplanMeier survival analysis with the log-rank test. p-values 
Table 1. Characteristics of AAV patients at diagnosis and during follow-up $(n=101)$

\begin{tabular}{|c|c|}
\hline AAV patients & Values \\
\hline \multicolumn{2}{|l|}{ At the time of diagnosis } \\
\hline \multicolumn{2}{|l|}{ Demographic data } \\
\hline Age (yr) & $62.0(21.0)^{*}$ \\
\hline Male sex & $34(33.7)$ \\
\hline \multicolumn{2}{|l|}{ AAV subtypes } \\
\hline MPA & $56(55.4)$ \\
\hline GPA & $28(27.7)$ \\
\hline EGPA & $17(16.8)$ \\
\hline \multicolumn{2}{|l|}{ ANCA positivity } \\
\hline MPO-ANCA (or P-ANCA) positivity & $73(72.3)$ \\
\hline PR3-ANCA (or C-ANCA) positivity & $18(17.8)$ \\
\hline Both ANCA positivity & $5(5.0)$ \\
\hline ANCA negativity & $15(14.9)$ \\
\hline \multicolumn{2}{|l|}{ AAV-specific indices } \\
\hline BVAS & $12.0(11.0)^{*}$ \\
\hline FFS & $1.0(1.0)^{*}$ \\
\hline \multicolumn{2}{|l|}{ Clinical manifestations at diagnosis } \\
\hline General & $40(39.6)$ \\
\hline Cutaneous & $15(14.9)$ \\
\hline Muco-membranous /Ocular & $4(4.0)$ \\
\hline Ear nose throat & $48(47.5)$ \\
\hline Pulmonary & $67(66.3)$ \\
\hline Cardiovascular & $11(10.9)$ \\
\hline Gastrointestinal & $4(4.0)$ \\
\hline Renal & $61(60.4)$ \\
\hline Nervous & $25(24.8)$ \\
\hline \multicolumn{2}{|l|}{ Routine laboratory results } \\
\hline Urine protein creatinine ratio & $1.42(2.69)^{*}$ \\
\hline Proteinuria & $42(41.5)$ \\
\hline Hematuria & $49(48.5)$ \\
\hline White blood cell count $\left(/ \mathrm{mm}^{3}\right)$ & $8,910.0(5,857.5)^{*}$ \\
\hline Haemoglobin (g/dL) & $11.3(3.5)^{*}$ \\
\hline Platelet count $\left(\times 1,000 / \mathrm{mm}^{3}\right)$ & $307.5(178.0)^{*}$ \\
\hline Fasting glucose (mg/dL) & $103.0(28.5)^{*}$ \\
\hline Blood urea nitrogen $(\mathrm{mg} / \mathrm{dL})$ & $17.9(22.8)^{*}$ \\
\hline Serum creatinine (mg/dL) & $0.9(1.8)^{*}$ \\
\hline Total protein $(\mathrm{g} / \mathrm{dL})$ & $6.8(1.2)^{*}$ \\
\hline Serum albumin (g/dL) & $3.7(1.0)^{*}$ \\
\hline Alkaline phosphatase (IU/L) & $74.0(35.8)^{*}$ \\
\hline Aspartate aminotransferase (IU/L) & $18.0(7.0)^{*}$ \\
\hline Alanine aminotransferase (IU/L) & $15.0(13.3)^{*}$ \\
\hline Total bilirubin (mg/dL) & $0.5(0.4)^{*}$ \\
\hline $\mathrm{ESR}(\mathrm{mm} / \mathrm{hr})$ & $63.5(68.3)^{*}$ \\
\hline $\mathrm{CRP}(\mathrm{mg} / \mathrm{L})$ & $8.0(69.1)^{*}$ \\
\hline \multicolumn{2}{|l|}{ Complement } \\
\hline $\mathrm{CH} 50(\mathrm{U} / \mathrm{mL})$ & $60.4(17.9)^{*}$ \\
\hline $\mathrm{C} 3$ (mg/dL) & $113.0(37.6)^{*}$ \\
\hline $\mathrm{C} 4(\mathrm{mg} / \mathrm{dL})$ & $25.6(12.4) *$ \\
\hline \multicolumn{2}{|l|}{ During the follow-up period } \\
\hline Follow-up duration (mo) & $26.4(30.4)^{*}$ \\
\hline
\end{tabular}

Table 1. Continued

\begin{tabular}{lc}
\hline \multicolumn{1}{c}{ AAV patients } & Values \\
\hline Poor outcomes during follow-up & \\
All-cause mortality & $9(8.9)$ \\
Duration until all-cause mortality (mo) & $26.4(30.4)^{*}$ \\
Relapse & $31(30.7)$ \\
Duration until relapse (mo) & $20.9(25.5)^{*}$ \\
ESRD & $20(19.8)$ \\
Duration until ESRD (mo) & $20.8(28.3)^{*}$ \\
Medications administered during the & \\
follow-up period & \\
Glucocorticoid & $96(95)$ \\
Cyclophosphamide & $52(51.5)$ \\
Rituximab & $19(18.8)$ \\
Azathioprine & $61(60.4)$ \\
Mycophenolate mofetil & $13(12.9)$ \\
Tacrolimus & $6(5.9)$ \\
Methotrexate & $9(8.9)$ \\
\hline
\end{tabular}

Values are expressed as the median (interquartile range) or number (\%). AAV: ANCA-associated vasculitis, ANCA: antineutrophil cytoplasmic antibody, MPA: microscopic polyangiitis, GPA: granulomatosis with polyangiitis, EGPA: eosinophilic GPA, MPO: myeloperoxidase, P: perinuclear, PR3: proteinase 3, C: cytoplasmic, BVAS: Birmingham Vasculitis Activity Score, FFS: Five-Factor score, ESR: erythrocyte sedimentation rate, CRP: C-reactive protein, $\mathrm{CH} 50$ : total haemolytic complement activity, C3: complement 3, C4: complement 4, ESRD: end-stage renal disease. *Median (interquartile range).

$<0.05$ were considered statistically significant.

\section{RESULTS}

\section{Characteristics of AAV patients at diagnosis and during follow-up}

At diagnosis, the median age of the $101 \mathrm{AAV}$ patients was 62.0 years, and 34 patients were men. MPA was the most common AAV subtype (55.4\%), and ANCA was positive in 86 patients. In addition, the median baseline BVAS and FFS were 12.0 and 1.0, respectively. Table 1 presents the results of routine laboratory tests. The median $\mathrm{CH} 50$ level was $60.4 \mathrm{U} / \mathrm{mL}$, and the median follow-up duration was 26.4 months. All-cause death was reported in 9 patients during follow-up, whereas relapse and ESRD developed in 31 and 20 patients, respectively.

\section{Correlation of continuous variables with $\mathrm{CH} 50$ at diagnosis \\ CH50 was significantly correlated with age and fasting}


glucose. Moreover, it was positively correlated with $\mathrm{C} 4$ $(r=0.584 ; p<0.001)$ but not $\mathrm{C} 3(\mathrm{r}=0.061 ; \mathrm{p}=0.555)$. Of note, $\mathrm{CH} 50$ was negatively correlated with the BVAS $(\mathrm{r}=$

Table 2. Correlation of continuous variables with the $\mathrm{CH} 50$ at diagnosis in AAV patients

\begin{tabular}{lrr}
\hline \multicolumn{1}{c}{ AAV patients } & $\begin{array}{c}\text { Correlation } \\
\text { coefficient }(\mathrm{r})\end{array}$ & $\mathrm{p}$-value \\
\hline Age $(\mathrm{yr})$ & 0.202 & 0.043 \\
BVAS & -0.241 & 0.015 \\
FFS & 0.030 & 0.762 \\
White blood cell count $\left(/ \mathrm{mm}^{3}\right)$ & -0.101 & 0.317 \\
Haemoglobin $(\mathrm{g} / \mathrm{dL})$ & -0.013 & 0.869 \\
Platelet count $\left(\times 1,000 / \mathrm{mm}^{3}\right)$ & -0.014 & 0.891 \\
Fasting glucose $(\mathrm{mg} / \mathrm{dL})$ & 0.299 & 0.002 \\
Blood urea nitrogen $(\mathrm{mg} / \mathrm{dL})$ & 0.083 & 0.408 \\
Serum creatinine $(\mathrm{mg} / \mathrm{dL})$ & 0.011 & 0.915 \\
Total protein $(\mathrm{g} / \mathrm{dL})$ & 0.102 & 0.308 \\
Serum albumin $(\mathrm{g} / \mathrm{dL})$ & 0.129 & 0.198 \\
Alkaline phosphatase $(\mathrm{IU} / \mathrm{L})$ & 0.013 & 0.900 \\
Aspartate aminotransferase $(\mathrm{IU} / \mathrm{L})$ & 0.039 & 0.699 \\
Alanine aminotransferase $(\mathrm{IU} / \mathrm{L})$ & 0.028 & 0.781 \\
Total bilirubin $(\mathrm{mg} / \mathrm{dL})$ & -0.003 & 0.974 \\
ESR (mm/hr) & 0.122 & 0.225 \\
CRP (mg/L) & -0.002 & 0.988 \\
C3 (mg/dL) & 0.061 & 0.555 \\
C4 (mg/dL) & 0.584 & $<0.001$ \\
\hline CH & & \\
\hline
\end{tabular}

CH50: total haemolytic complement activity, AAV: ANCAassociated vasculitis, ANCA: antineutrophil cytoplasmic antibody, BVAS: Birmingham Vasculitis Activity Score, FFS: Five-Factor score, ESR: erythrocyte sedimentation rate, CRP: C-reactive protein, C3: complement 3, C4: complement 4.

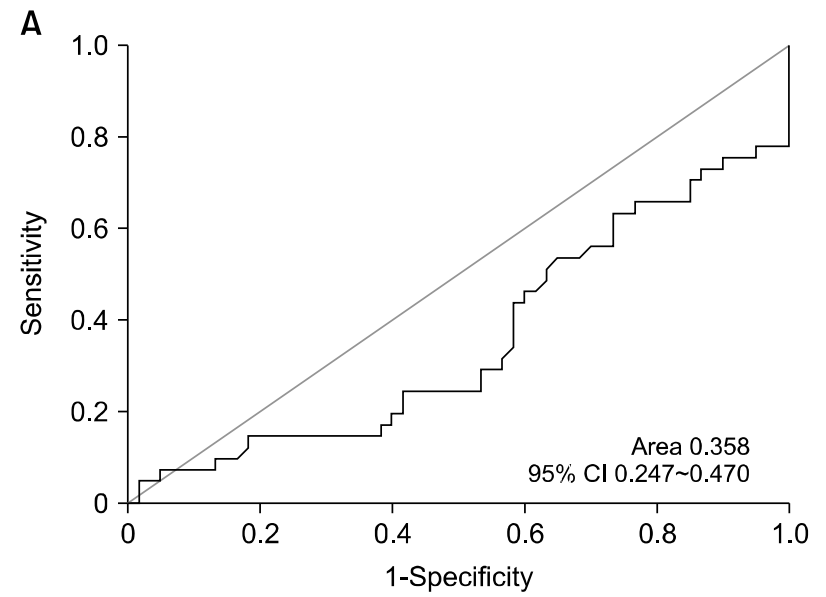

$-0.241 ; \mathrm{p}=0.015)$ (Table 2).

\section{Optimal cut-off of $\mathrm{CH} 50$ at diagnosis}

The optimal CH50 cut-off at diagnosis for the high baseline activity (based on the BVAS, BVAS $\geq 13$ ) was set below $62.1 \mathrm{U} / \mathrm{mL}$, at which the sensitivity was $53.3 \%$ and the specificity was $75.6 \%$ (area, 0.358 ; $95 \%$ confidence interval [CI], 0.247 to 0.470 ) (Figure 1A). When we classified the AAV patients into two groups based on the calculated CH50 cut-off, 59 of the 101 patients were separated into the low-CH50 group. The high baseline activity of AAV, based on the BVAS, was identified more frequently in the low-CH50 group than in the high-CH50 group ( $52.5 \%$ vs. $23.8 \%$; $\mathrm{p}=0.004$ ). Furthermore, the low-CH50 group had a significantly higher risk for the high baseline activity of AAV than the high-CH50 group (RR, 3.543; 95\% CI, 1.477 to 8.497) (Figure 1B).

\section{Linear regression analysis based on the BVAS at di- agnosis}

In the univariable linear regression analysis, $\mathrm{CH} 50$, white blood cell and platelet counts, haemoglobin, BUN, serum albumin, ALP, alanine aminotransferase, ESR, and CRP were significantly correlated with the BVAS at diagnosis. However, in the multivariable analysis, only CH50 ( $\beta=-0.265 ; 95 \% \mathrm{CI},-0.173$ to -0.036$)$ and haemoglobin ( $\beta=-0.374 ; 95 \% \mathrm{CI},-1.842$ to -0.261 ) were significantly associated with the BVAS at diagnosis (Table 3).

B

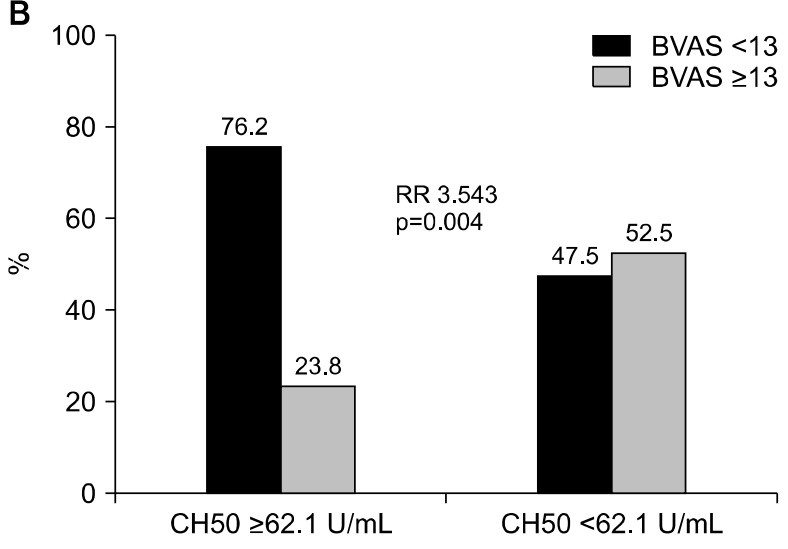

Figure 1. Optimal $\mathrm{CH} 50$ cut-off and relative risk. When the optimal $\mathrm{CH} 50$ cut-off at diagnosis for the high baseline activity of AAV based on the BVAS (BVAS $\geq 13$ ) was set below $62.1 \mathrm{U} / \mathrm{m}$, AAV patients with $\mathrm{CH} 50<62.1 \mathrm{U} / \mathrm{mL}$ had a significantly higher risk for a high baseline activity of AAV based on the BVAS than those with $\mathrm{CH} 50 \geq 62.1 \mathrm{U} / \mathrm{mL}$. $\mathrm{CH} 50$ : total haemolytic complement activity, BVAS: Birmingham Vasculitis Activity Score, AAV: antineutrophil cytoplasmic antibody-associated vasculitis, Cl: confidence interval, RR: relative risk. 
Table 3. Linear regression of continuous variables based on the BVAS in AAV patients

\begin{tabular}{|c|c|c|c|c|c|}
\hline \multirow[b]{2}{*}{ Variables } & \multicolumn{2}{|c|}{ Univariable } & \multicolumn{3}{|c|}{ Multivariable } \\
\hline & $\begin{array}{c}\text { Standardised } \\
\text { correlation } \\
\text { coefficient }(\beta)\end{array}$ & p-value & $\begin{array}{l}\text { Standardised } \\
\text { correlation } \\
\text { coefficient }(\beta)\end{array}$ & $\begin{array}{c}95 \% \text { confidence } \\
\text { interval }\end{array}$ & p-value \\
\hline $\mathrm{CH} 50(\mathrm{U} / \mathrm{mL})$ & -0.241 & 0.015 & -0.265 & -0.173 to -0.036 & 0.003 \\
\hline $\mathrm{C} 3(\mathrm{mg} / \mathrm{dL})$ & -0.015 & 0.884 & & & \\
\hline $\mathrm{C} 4(\mathrm{mg} / \mathrm{dL})$ & -0.140 & 0.175 & & & \\
\hline Age (yr) & 0.101 & 0.314 & & & \\
\hline White blood cell count $\left(/ \mathrm{mm}^{3}\right)$ & 0.198 & 0.047 & 0.032 & 0.000 to 0.000 & 0.791 \\
\hline Haemoglobin (g/dL) & -0.500 & $<0.001$ & -0.374 & -1.842 to -0.261 & 0.010 \\
\hline Platelet count $\left(\times 1,000 / \mathrm{mm}^{3}\right)$ & 0.262 & 0.008 & 0.106 & -0.006 to 0.015 & 0.402 \\
\hline Fasting glucose (mg/dL) & -0.033 & 0.741 & & & \\
\hline Blood urea nitrogen (mg/dL) & 0.286 & 0.004 & 0.059 & -0.054 to 0.098 & 0.575 \\
\hline Serum creatinine (mg/dL) & 0.179 & 0.074 & & & \\
\hline Total protein (g/dL) & 0.035 & 0.731 & & & \\
\hline Serum albumin (g/dL) & -0.440 & $<0.001$ & -0.021 & -2.951 to 2.591 & 0.898 \\
\hline Alkaline phosphatase (IU/L) & 0.225 & 0.025 & 0.185 & -0.004 to 0.061 & 0.084 \\
\hline Aspartate aminotransferase (IU/L) & -0.120 & 0.234 & & & \\
\hline Alanine aminotransferase (IU/L) & -0.228 & 0.022 & -0.093 & -0.116 to 0.047 & 0.399 \\
\hline Total bilirubin (mg/dL) & -0.151 & 0.135 & & & \\
\hline $\mathrm{ESR}(\mathrm{mm} / \mathrm{hr})$ & 0.327 & 0.001 & 0.175 & -0.014 to 0.075 & 0.180 \\
\hline $\mathrm{CRP}(\mathrm{mg} / \mathrm{L})$ & 0.237 & 0.017 & -0.132 & -0.049 to 0.020 & 0.401 \\
\hline
\end{tabular}

BVAS: Birmingham Vasculitis Activity Score, AAV: ANCA-associated vasculitis, ANCA: antineutrophil cytoplasmic antibody, $\mathrm{CH} 50$ : total haemolytic complement activity, C3: complement 3, C4: complement 4, ESR: erythrocyte sedimentation rate, CRP: C-reactive protein.

\section{Poor-outcome-free survival rates during follow-up} With regard to poor outcomes, the low-CH50 group exhibited a lower relapse-free survival rate than the highCH50 group; however, this difference did not reach significance $(p=0.082)$. Additionally, there were no significant differences in the ESRD-free survival rate between the two groups (Figure 2).

\section{Association between hypocomplementemia and baseline high disease activity and poor-outcomes} We analyzed whether disease activity was associated with hypocomplementemia, defined as $\mathrm{CH} 50, \mathrm{C} 3$, or $\mathrm{C} 3$ below the reference range. AAV patients with hypocomplementemia showed a significantly higher risk of high baseline activity of AAV, based on the BVAS, than those without hypocomplementemia (RR, 8.821; 95\% CI, 2.323 33.501) (Figure 3). In addition, regarding poor outcomes during follow-up, AAV patients with hypocomplementemia exhibited a significantly lower $\mathrm{cu}-$ mulative ESRD-free survival rate than those without hypocomplementemia $(p=0.002)$. However, no significant differences were observed in both the cumulative pa- tients' overall and relapse-free survival rates between the groups ( $\mathrm{p}=0.767$ and 0.055 , respectively) (Figure 4 ).

\section{DISCUSSION}

In this study, we investigated whether $\mathrm{CH} 50$ levels at diagnosis could reflect the baseline activity and predict the prognosis of AAV. At the diagnosis of AAV, $\mathrm{CH} 50$ was inversely correlated with the BVAS, and based on our data, the suggested cut-off $\mathrm{CH} 50$ level to predict severe disease of AAV was $62.1 \mathrm{U} / \mathrm{mL}$. Summarising the results of this study, the following conclusions were drawn: the low $\mathrm{CH} 50$ level at diagnosis was associated with severe disease of AAV; however, it did not predict the occurrence of poor outcomes of AAV.

Among C3 and C4, only C4 positively correlated with $\mathrm{CH} 50$, which primarily reflected the activation of the classical complement pathway. At the beginning of this study, C3 was also expected to be well-correlated with $\mathrm{CH} 50$ because the alternative complement pathway is known to be mainly involved in the pathophysiology of AAV compared with the classical complement pathway. However, no cor- 

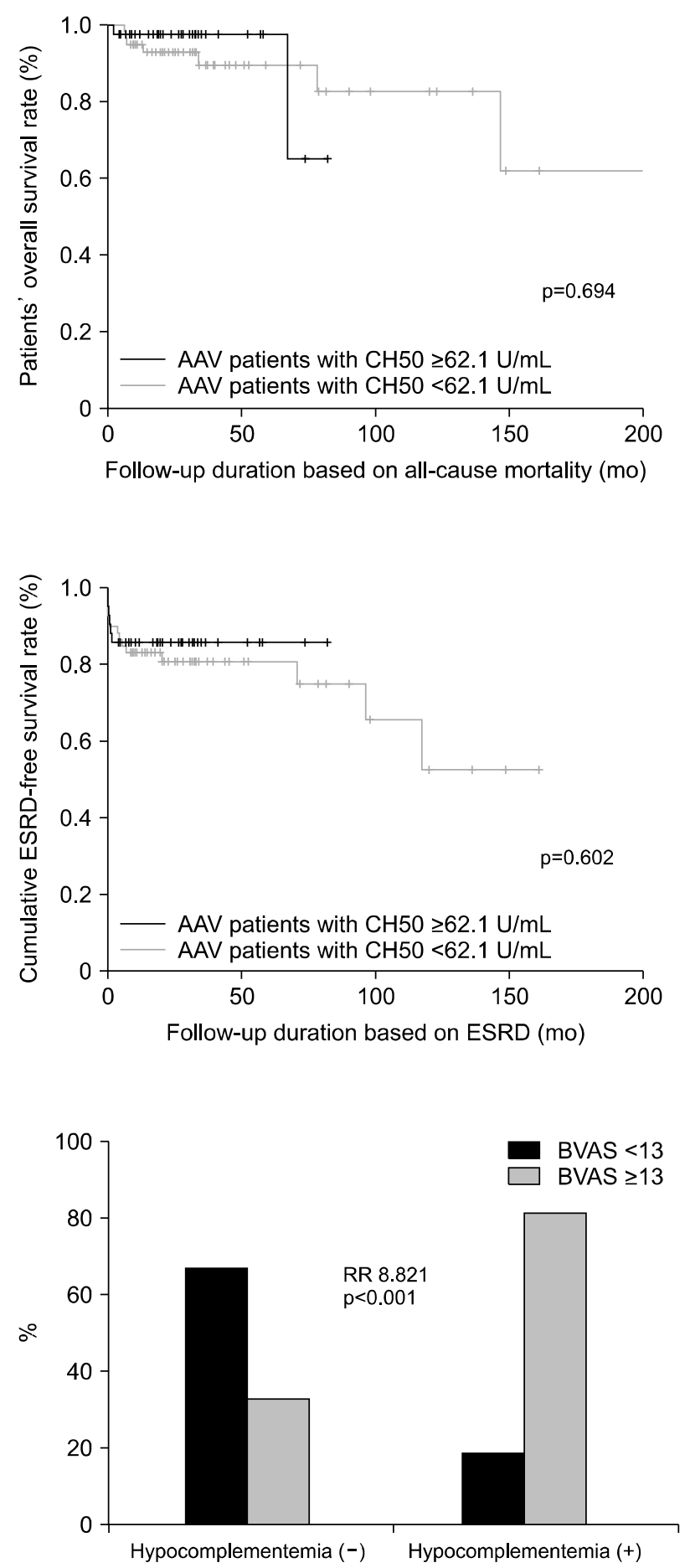

Figure 3. Relative risk of high disease activity regarding hypocomplementemia. AAV patients with hypocomplementemia, defined as below reference range of $\mathrm{CH} 50, \mathrm{C} 3$, or $\mathrm{C} 4$, showed significantly higher risk for a high baseline activity of AAV (BVAS $\geq 13$ ). CH50: total haemolytic complement activity, AAV: antineutrophil cytoplasmic antibody-associated vasculitis, BVAS: Birmingham Vasculitis Activity Score.

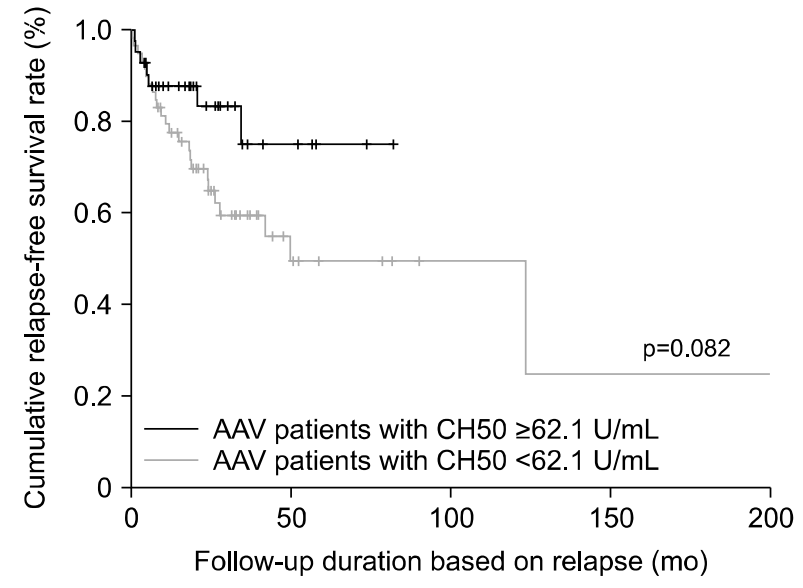

Figure 2. Comparison of poor-outcome-free survival rates. There were no significant differences in the relapse-free and ESRD-free survival rates between the AAV patients with $\mathrm{CH} 50<62.1 \mathrm{U} / \mathrm{mL}$ and those with $\mathrm{CH} 50 \geq 62.1 \mathrm{U} / \mathrm{mL}$. $\mathrm{CH} 50$ : total haemolytic complement activity, ESRD: end-stage renal disease, AAV: antineutrophil cytoplasmic antibody-associated vasculitis.

relation was found between $\mathrm{CH} 50$ and $\mathrm{C} 3$. Therefore, to validate this finding, a method of interpreting "which complement pathway is activated' was adopted, based on the patterns of increases and decreases in $\mathrm{CH} 50, \mathrm{C} 3$, and $\mathrm{C} 4$ levels. In this method, when $\mathrm{CH} 50$ and $\mathrm{C} 4$ levels were reduced, regardless of $\mathrm{C} 3$ levels, we found that the classical complement pathway was predominantly involved. Concurrently, when $\mathrm{CH} 50$ and $\mathrm{C} 3$ levels were reduced and the $\mathrm{C} 4$ level was normal, we considered that the alternative complement pathway was more frequently involved [15]. In the present study, 6 out of 101 patients had a $\mathrm{CH} 50$ level below the normal reference range according to the haemolysis method, and all these patients exhibited a high baseline activity of AAV based on the BVAS. Furthermore, three of the six patients with a $\mathrm{CH} 50$ level below the normal reference range exhibited low $\mathrm{C} 3$ or C4. One patient showed low C3, which indicated alternative complement pathway activation. However, another patient demonstrated low $\mathrm{C} 4$, and the third patient exhibited both low C3 and C4, which suggested a classical 

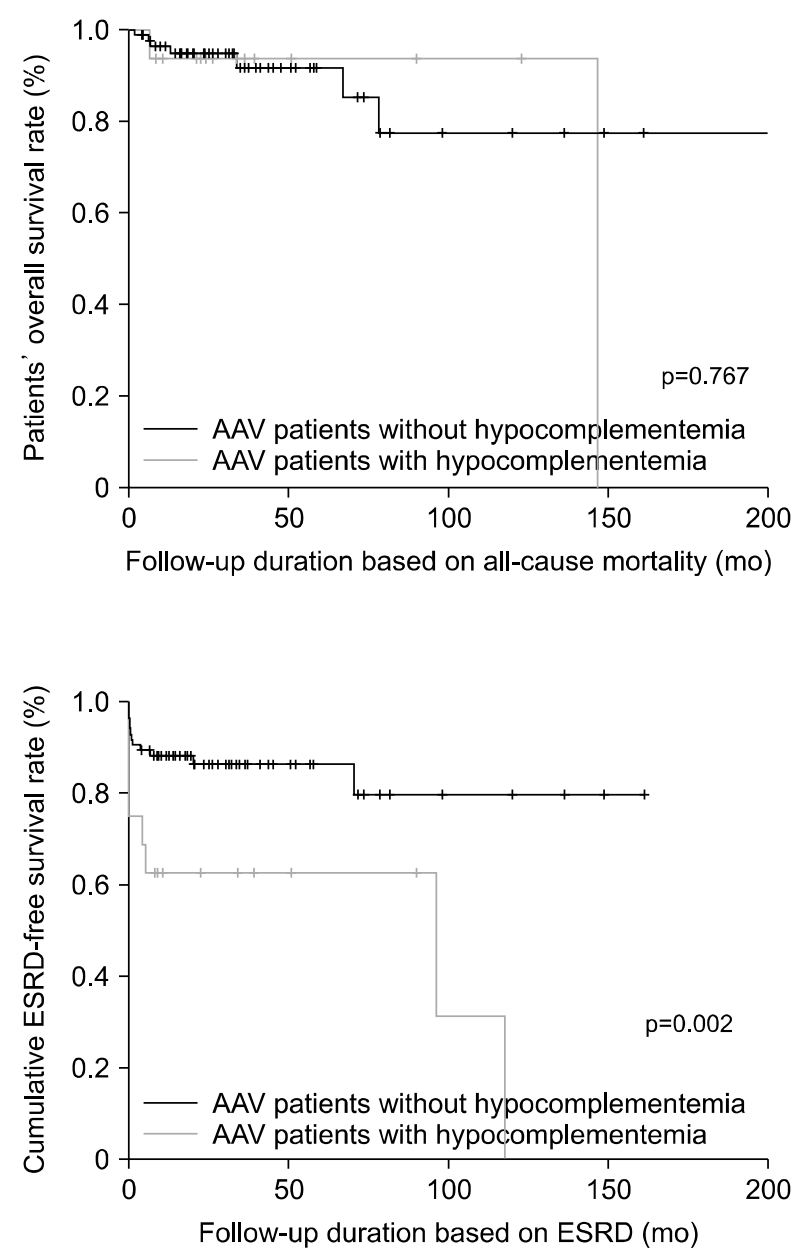

complement pathway activation. With these results, we could not conclude which complement pathway was predominantly contributing to the high baseline activity of AAV.

Fukui et al. [9] reported that hypocomplementemia, which was defined as a low $\mathrm{CH} 50, \mathrm{C} 3$, or C4 level, was associated with death and organ damage in patients with AAV. In this study, we investigated whether hypocomplementemia could reflect the high baseline activity of AAV and predict poor outcomes. Our data showed that AAV patients with hypocomplementemia showed a significantly higher risk of high baseline activity (based on the BVAS, BVAS $\geq 13$ ) and significantly lower cumulative ESRD-free survival than those without hypocomplementemia. However, unlike that in the previous study [9], no significant differences were observed in both the cumulative patients' overall and relapse-free survival rates between the groups. Further studies are needed to analyze these discrepancies and conclude an association between hypocomplementemia and disease activity and poor outcomes

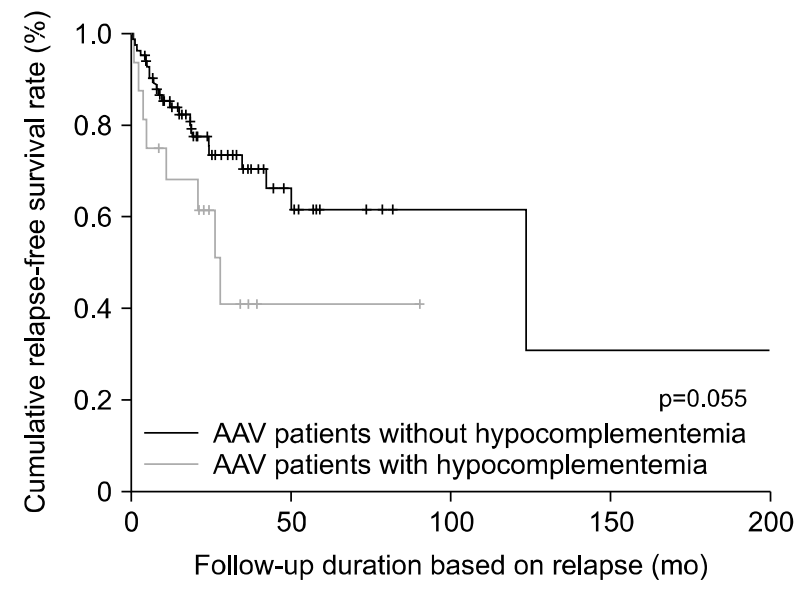

Figure 4. Comparison of poor-outcome-free survival rates regarding hypocomplementemia. There were no significant differences in the cumulative patients' overall and relapse-free survival rates between the AAV patients with or without hypocomplementemia. However, AAV patients with hypocomplementemia exhibited a significantly lower cumulative ESRD-free survival rate than those without hypocomplementemia. AAV: antineutrophil cytoplasmic antibody-associated vasculitis, ESRD: end-stage renal disease.

of AAV. Moreover, we examined whether low C3 or low $\mathrm{C} 4$ could reflect the baseline activity of AAV and predict ESRD occurrence (data not presented). We found that $A A V$ patients with low $C 3$ exhibited a significantly higher risk of high baseline activity of AAV, based on the BVAS, than those with normal C3 levels (RR, 5.129; 95\% CI, $1.291 \sim 20.382$ ). Furthermore, a low $\mathrm{C} 3$ value at diagnosis had the predictive potential for ESRD occurrence during follow-up, compared with a normal C3 ( $\mathrm{p}=0.001)$. Conversely, a low $\mathrm{C} 4$ value at diagnosis could neither reflect a high baseline activity of AAV based on the BVAS nor predict the occurrence of ESRD during follow-up in AAV patients $(\mathrm{p}=0.069$ and 0.061 , respectively). Consequently, we conclude that low $\mathrm{CH} 50$ and low $\mathrm{C} 3$ among patients with hypocomplementemia reflect the baseline activity, whereas only low C3 predicts ESRD occurrence during follow-up.

In this study, we used a $\mathrm{CH} 50$ value of $61.2 \mathrm{U} / \mathrm{mL}$, rather than the reference range, as the optimal cut-off for the high baseline activity of AAV. In the univariable analysis, 
at diagnosis, both $\mathrm{CH} 50<62.1 \mathrm{U} / \mathrm{mL}$ (OR, 3.543; 95\% CI, $1.477 \sim 8.497$ ) and low C3 (OR, 5.129; 95\% CI , 1.291 $\sim 20.382$ ) were significantly associated with the high baseline activity of AAV based on the BVAS. However, in the multivariable analysis with two variables, $\mathrm{CH} 50$ $<62.1 \mathrm{U} / \mathrm{mL}$ (OR, 3.320; 95\% CI, 1.327 8.306) was significantly associated with high baseline activity of AAV; however, low C3 (OR, 3.389; 95\% CI, 0.812 12.147) was not. Therefore, $\mathrm{CH} 50$ below the optimal cut-off is more efficient than low $\mathrm{C} 3$, reflecting the high baseline activity of AAV based on the BVAS; however, for predicting poor renal outcomes, low $\mathrm{C} 3$ at diagnosis is better than $\mathrm{CH} 50$ below the optimal cut-off at diagnosis. A notable strength of this study is that we provided a novel cut-off value for $\mathrm{CH} 50$, which was correlated with baseline disease activity. Furthermore, our patient population was immunosuppressant-naïve and diagnosed at a single centre, which can minimise the confounding factor. However, our study had the following limitations. First, the number of study subjects was not large enough to generalise and apply the results of our study to all patients with AAV. Second, because we do not have separate validation group, the cut-off value of $\mathrm{CH} 50$ as $61.2 \mathrm{U} / \mathrm{mL}$ should be validated in other AAV cohorts in future studies. In addition, it was conducted as a retrospective study. Hence, alternative complement pathway activation (AH50) and Factor B could not be evaluated in this study. Inclusion of AH50 and Factor B might have enabled a more accurate interpretation of the $\mathrm{CH} 50, \mathrm{C} 3$, and $\mathrm{C} 4$ patterns $[5,15]$.

\section{CONCLUSION}

CH50 levels may reflect the baseline disease activity in AAV patients. Future studies with a larger patient population may provide more reliable data regarding disease prognosis associated with $\mathrm{CH} 50$, as there was a tendency toward an association between $\mathrm{CH} 50$ and the relapse-free survival rate in this study.

\section{ACKNOWLEDGMENTS}

This study was supported by a faculty research grant of Yonsei University College of Medicine (6-2019-0184) and a grant from the Korea Health Technology R\&D Project through the Korea Health Industry Development Institute, funded by the Ministry of Health and Welfare, Republic of Korea (HI14C1324).

\section{CONFLICT OF INTEREST}

No potential conflict of interest relevant to this article was reported.

\section{AUTHOR CONTRIBUTIONS}

Design of this study: S.W.L. Interpretation and statistical analysis of data: J.Y.P., L.E.L., and S.W.L. Collection of data: J.Y.P., L.E.L., S.S.A., and S.W.L. Drafting the manuscript: J.Y.P. and S.W.L. Approval of final manuscript: J.Y.P., L.E.L., S.S.A., J.J.S., Y.B.P., and S.W.L.

\section{REFERENCES}

1. Jennette JC, Falk RJ, Bacon PA, Basu N, Cid MC, Ferrario F, et al. 2012 revised International Chapel Hill Consensus Conference Nomenclature of Vasculitides. Arthritis Rheum 2013;65:1-11.

2. Watts R, Lane S, Hanslik T, Hauser T, Hellmich B, Koldingsnes W, et al. Development and validation of a consensus methodology for the classification of the ANCA-associated vasculitides and polyarteritis nodosa for epidemiological studies. Ann Rheum Dis 2007;66:222-7.

3. Jennette JC, Falk RJ. Pathogenesis of antineutrophil cytoplasmic autoantibody-mediated disease. Nat Rev Rheumatol 2014;10:463-73.

4. Lamprecht P, Kerstein A, Klapa S, Schinke S, Karsten CM, $\mathrm{Yu} \mathrm{X}$, et al. Pathogenetic and clinical aspects of anti-neutrophil cytoplasmic autoantibody-associated vasculitides. Front Immunol 2018;9:680.

5. Angioi A, Fervenza FC, Sethi S, Zhang Y, Smith RJ, Murray D, et al. Diagnosis of complement alternative pathway disorders. Kidney Int 2016;89:278-88.

6. Jennette JC, Falk RJ, Hu P, Xiao H. Pathogenesis of antineutrophil cytoplasmic autoantibody-associated small-vessel vasculitis. Annu Rev Pathol 2013;8:139-60.

7. Smith JG Jr. Vasculitis. J Dermatol 1995;22:812-22.

8. Kawakami T, Kimura S, Takeuchi S, Soma Y. Relationship among antineutrophil cytoplasmic antibody, blood urea nitrogen and complement in patients with eosinophilic granulomatosis polyangiitis (Churg-Strauss syndrome). J Dermatol 2013;40:511-5.

9. Fukui S, Iwamoto N, Umeda M, Nishino A, Nakashima Y, Koga T, et al. Antineutrophilic cytoplasmic antibody-associated vasculitis with hypocomplementemia has a higher incidence of serious organ damage and a poor prognosis. Medicine (Baltimore) 2016;95:e4871.

10. Molad Y, Tovar A, Ofer-Shiber S. Association of low serum complement $\mathrm{C} 3$ with reduced patient and renal survival in antimyeloperoxidase-associated small-vessel vasculitis. Nephron Clin Pract 2014;126:67-74.

11. Mukhtyar C, Lee R, Brown D, Carruthers D, Dasgupta B, Dubey $S$, et al. Modification and validation of the Birmingham Vasculitis Activity Score (version 3). Ann Rheum Dis 2009;68:1827-32. 
12. Guillevin L, Pagnoux C, Seror R, Mahr A, Mouthon L, Toumelin PL; French Vasculitis Study Group (FVSG). The five-factor score revisited: assessment of prognoses of systemic necrotizing vasculitides based on the French Vasculitis Study Group (FVSG) cohort. Medicine (Baltimore) 2011;90: 19-27.

13. McAdoo SP, Medjeral-Thomas N, Gopaluni S, Tanna A, Mansfield N, Galliford J, et al. Long-term follow-up of a combined rituximab and cyclophosphamide regimen in re- nal anti-neutrophil cytoplasm antibody-associated vasculitis. Nephrol Dial Transplant 2019;34:63-73.

14. Yamamoto S, Kubotsu K, Kida M, Kondo K, Matsuura S, Uchiyama S, et al. Automated homogeneous liposome-based assay system for total complement activity. Clin Chem 1995;41:586-90.

15. Ling M, Murali M. Analysis of the complement system in the clinical immunology laboratory. Clin Lab Med 2019;39: 579-90. 